\title{
Effects of The Long-Time Wearing of Gloves by Health Care Workers Managing Covid-19 Assessed by Electrodermal Activity: A Pilot Study
}

\author{
https://doi.org/10.3991/ijoe.v17i12.23439 \\ Haval Y. Yacoob Aldosky ${ }^{1(凶)}$, Dindar S. Bari², Ørjan G. Martinsen ${ }^{3}$ \\ ${ }^{1}$ University of Duhok, Duhok, Kurdistan Region, Iraq \\ ${ }^{2}$ University of Zakho, Zakho, Kurdistan Region, Iraq \\ ${ }^{3}$ University of Oslo, Oslo, Norway \\ yacoobaldosky@uod.ac
}

\begin{abstract}
Continues wearing gloves is a crucial problem among health care workers treating patients with coronavirus (COVID-19) infection that may cause skin complications. In the present study, the skin conductance (SC), skin potential (SP) and skin susceptance (SS) were used to identify the effects of long-term wearing gloves among health care workers managing COVID-19. The experimental procedure was repeated 30 times on three subjects recruited as volunteers to wear gloves for 4 hours. The baseline of parameters SC, SP, and SS were recorded at normal conditions without wearing the glove at zero time (To). The parameters were again recorded in the cases of glove-wearing with and without zinc oxide ointment $(\mathrm{ZnO})$ for the same period. Noticeable differences were found in each of SC, SP and SS for subjects who wore gloves when compared to the baseline for $4 \mathrm{~h}$. Results also showed significant differences in each of SP and SS during the same period with applying $\mathrm{ZnO}$ comparing to wearing gloves without applying $\mathrm{ZnO}$. The results show that skin hydration is significantly improved by using $\mathrm{ZnO}$ and changing gloves at least every four hours of continuous wearing is recommended. However, due to the small sample size and the specific study population, the results cannot be generalized to all ages of health workers.
\end{abstract}

Keywords - COVID-19, electrodermal activity, health care workers, wearing gloves

\section{Introduction}

Health professions preventing and controlling Coronavirus Disease 2019 (COVID-19) are prone to skin and mucous membrane injury, which may cause acute and chronic dermatitis, secondary infection, and aggravation of underlying skin diseases [1]. Previous studies have revealed that hand eczema is quite common in health care workers and the risk factors include frequent hand hygiene and wearing gloves for a long time. Long-term use of gloves may lead to overhydration of the stratum 
corneum, which may cause maceration and erosion [2]. It is axiomatic that gloves fuel a feeling deep within health care workers of self-protection. However, the major problem is that this feeling of extra protection causes them to wear gloves for longer than they should and perform hand hygiene less frequently than they should. Moreover, a study indicated that applying hand cream with long-term use of gloves could reverse maceration [1]. According to the survey, $56.7 \%$ of health care workers (HCWs) wore personal protective equipment (PPE) includes gloves for over 6 hours per day. As far as we know, no previous research has identified the effects of long-term wearing gloves to prevent a potential skin problem among health care workers managing COVID-19. One of the most accurate techniques that determine the skin hydration condition is electrodermal activity (EDA). EDA refers to the changes in the electrical properties of the skin as a result of sweat secretion. The EDA parameters, skin conductance (SC), skin potential (SP), are associated with the sweat ducts filling and sweat reabsorption, whereas skin susceptance (SS) is related to the moisture content of the stratum corneum (the outermost layer of the skin) [3],[4]. EDA is utilized within various fields such as in the fields of psychophysiology, anesthesia, neurology, and also, they are used for stress detection, sweating, and skin hydration estimations [4].

This study aims to estimate the effects of wearing gloves for a long duration for health care workers managing COVID-19 in two different situations (with and without Zinc Oxide Ointment $\mathrm{ZnO}$ ) depending on the skin hydration assessing by SC, SS and SP.

\section{$2 \quad$ Materials and methods}

\subsection{Instrumentation}

In this study, the three-electrode setup was employed [3]. The system consisted of a small front-end electronic box connected to a PC laptop via a National Instruments DAQ card- NI USB-6211 and running custom-written software in LabVIEW, version 14, analogous to the system presented in Bari et al. [3]. This computerized system enables simultaneous measurement of SC, SP, and SS at the same skin site.

\subsection{Experimental protocol and participants}

Due to the coronavirus (COVID-19) outbreak, and the restrictions imposed on access to the health centers in order to stop the spread of the virus, the test was limited to three participants with replicated procedures. EDA parameters were measured from three subjects apparently healthy, two males and one female with the average age of $33 \pm 3.46$ years using KittyCat hydrogel electrodes (Kendall, KittyCat ${ }^{\circledR}, 1050$ NPSM). During the data collection, they were sitting isolated and comfortable in a chair in a sound-free room with a temperature-control $\left(22-23^{\circ} \mathrm{C}\right)$. Before measurement, 5 min were allowed for the stabilization of electrodes after electrode application on one of the participants' hands, then SC, SP, and SS were measured. Measurements were repeated 10 times for each participant over a period of two weeks, with all environmental conditions constant.

These measurements were taken at three different cases as follows. 


\subsection{Baseline (To)}

In this experiment, $\mathrm{SC}, \mathrm{SP}$, and SS were recorded at normal conditions, i.e., without wearing the glove at zero time (To).

\subsection{Wearing the glove for 4 hours}

In this experiment, the same volunteers repeated the test, but with wearing the glove for 4 hours (working for a half day). Throughout the test, the subjects were wearing the glove on only one forearm (experimental hand), and were not allowed to remove or change the glove for this period to macerate (hydrate) the hand. Then the glove was removed and electrodes were connected to the experimental hand of the participants for EDA measurements.

\subsection{Treatment with zinc oxide and wearing the glove for 4 hours}

In this experiment, again the same volunteers were asked to repeat the test, but before wearing the glove their experimental hands were treated with $\mathrm{ZnO}(15 \%)$ skin protectant to protect hands from irritants, then they wore the glove. After 4 hours, the glove was removed and electrodes were connected for EDA measurements.

The results of the three experiments were collected for each of electrical conductivity, potential, and subjects' sensitivity as a function of glove wearing time.

\subsection{Statistical analysis}

Descriptive and statistical analyses were performed with SPSS (IBM 22) for all parameters using a paired T-test. A p-values of less than 0.05 were accepted as significant.

\section{Results}

Descriptive analyses were performed, and values are displayed as means, standard deviations and confidence interval $95 \%$ as shown in Table 1 . The average of the EDA (SC, SS, and SP) parameters extracted for the three participants are illustrated in Figures 1, 2, and 3. Figures 1 and 2 show that wearing gloves for 4 hours without a $\mathrm{ZnO}$ led to an increase in SC, significant increase in SS, and negatively increased SP compared to control at baseline. $\mathrm{ZnO}$ application, on the contrary, reduced SC, slight decrease in the median value of SS, and negatively significant decrease in SP compared to wearing gloves without $\mathrm{ZnO}$ as shown in Figures 1, 2, and 3 respectively. No significant difference was observed in SS between wearing gloves with and without $\mathrm{ZnO}$. 
Table 1. Descriptive and statistical analyses for the EDA parameters

\begin{tabular}{|c|c|c|c|c|c|c|}
\hline \multirow{2}{*}{ Parameters } & \multirow{2}{*}{$\begin{array}{c}\text { Time of Wearing } \\
\text { The Glove (hr.) }\end{array}$} & \multirow{2}{*}{ Mean } & \multirow{2}{*}{$S D$} & \multicolumn{2}{|c|}{$95 \% C I$} & \multirow{2}{*}{ P-value } \\
\hline & & & & Lower & Upper & \\
\hline \multirow[t]{3}{*}{$\mathrm{SC}(\mu \mathrm{S})$} & Baseline $\left(\mathrm{T}_{0}\right)$ & 36.75 & 5.86 & 18.11 & 55.39 & $N S$ \\
\hline & $4 \mathrm{hr}$ & 43.96 & 6.12 & 24.47 & 63.44 & $N S$ \\
\hline & 4 hr. with $(\mathrm{ZnO})$ & 24.54 & 3.91 & 12.11 & 36.97 & $N S$ \\
\hline \multirow[t]{3}{*}{$\mathrm{SS}(\mu \mathrm{S})$} & Baseline $\left(\mathrm{T}_{0}\right)$ & 6.94 & 1.14 & 3.30 & 10.59 & \multirow[t]{2}{*}{$<0.05^{\mathrm{a}}$} \\
\hline & $4 \mathrm{hr}$. & 11.56 & 2.44 & 3.76 & 19.35 & \\
\hline & 4 hr with $(\mathrm{ZnO})$ & 10.89 & 1.60 & 5.79 & 15.98 & $N S$ \\
\hline \multirow[t]{3}{*}{$\mathrm{SP}(\mathrm{mV})$} & Baseline $\left(\mathrm{T}_{0}\right)$ & -11.99 & 2.45 & -19.81 & -4.17 & $N S$ \\
\hline & $4 \mathrm{hr}$ & -15.61 & 2.98 & -25.12 & -6.10 & \multirow[t]{2}{*}{$<0.05^{\mathrm{b}}$} \\
\hline & $4 \mathrm{hr}$ with $(\mathrm{ZnO})$ & -11.72 & 3.02 & -21.35 & -2.10 & \\
\hline
\end{tabular}

Notes: SC, Skin Conductance; SS, Skin Susceptance; SP, Skin Potential; SD, standard deviation; a Significant difference in Skin Susceptance between Baseline $\left(\mathrm{T}_{\mathrm{o}}\right)$ and $4 \mathrm{hr}$; ${ }^{\mathrm{b}}$ Significant difference in Skin Potential between $4 \mathrm{hr}$ with and without $\mathrm{ZnO}, N S$, Non-Significant.

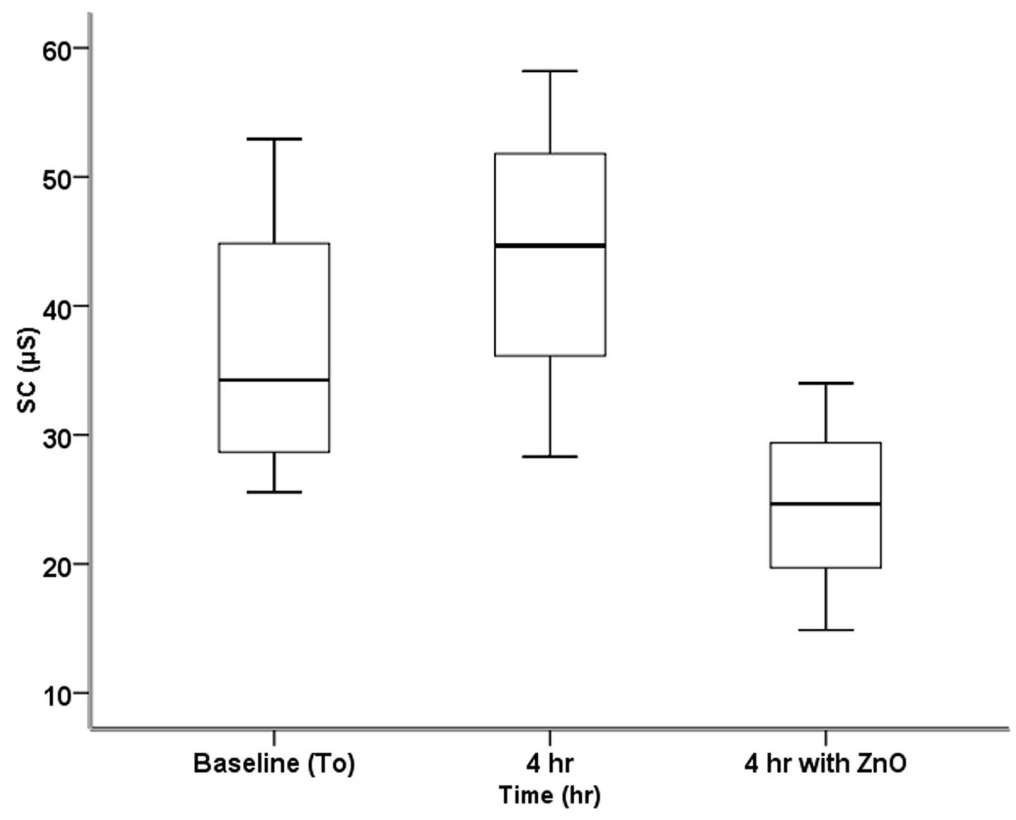

Fig. 1. Impact of wearing gloves for a long duration with and without $\mathrm{ZnO}$ on skin conductance (SC) 


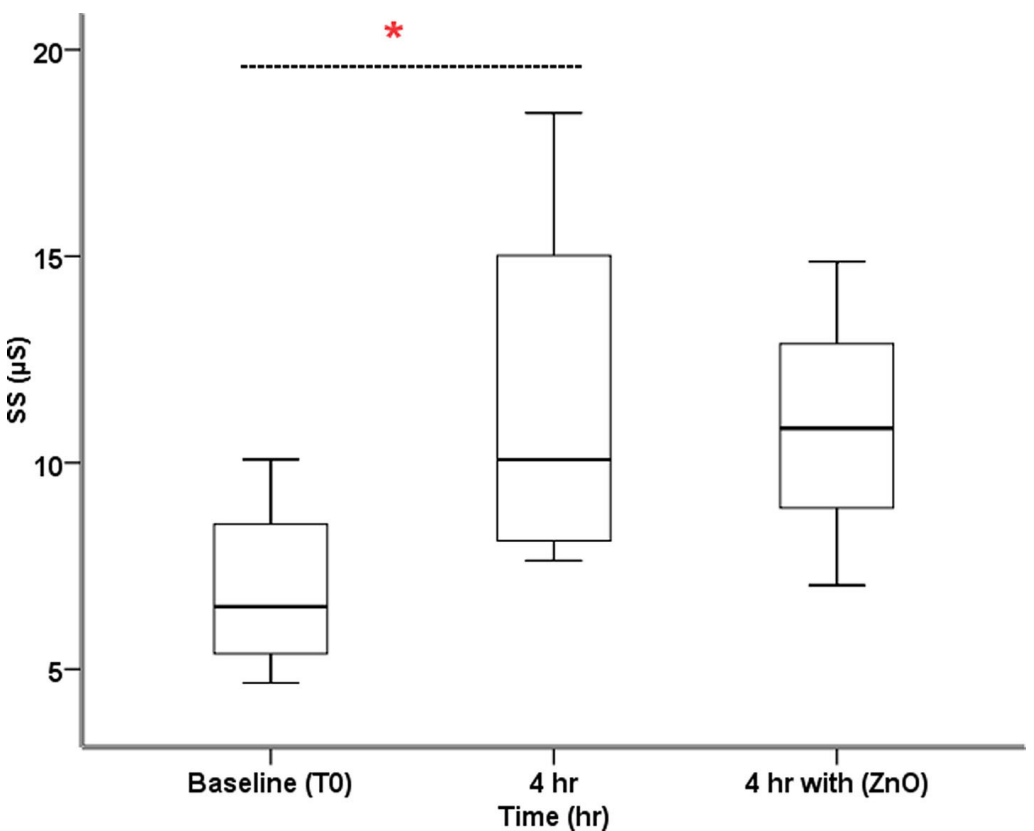

Fig. 2. Impact of wearing gloves for a long duration with and without $\mathrm{ZnO}$ on skin susceptance (SS). ${ }^{*} \mathrm{p}<0.05$

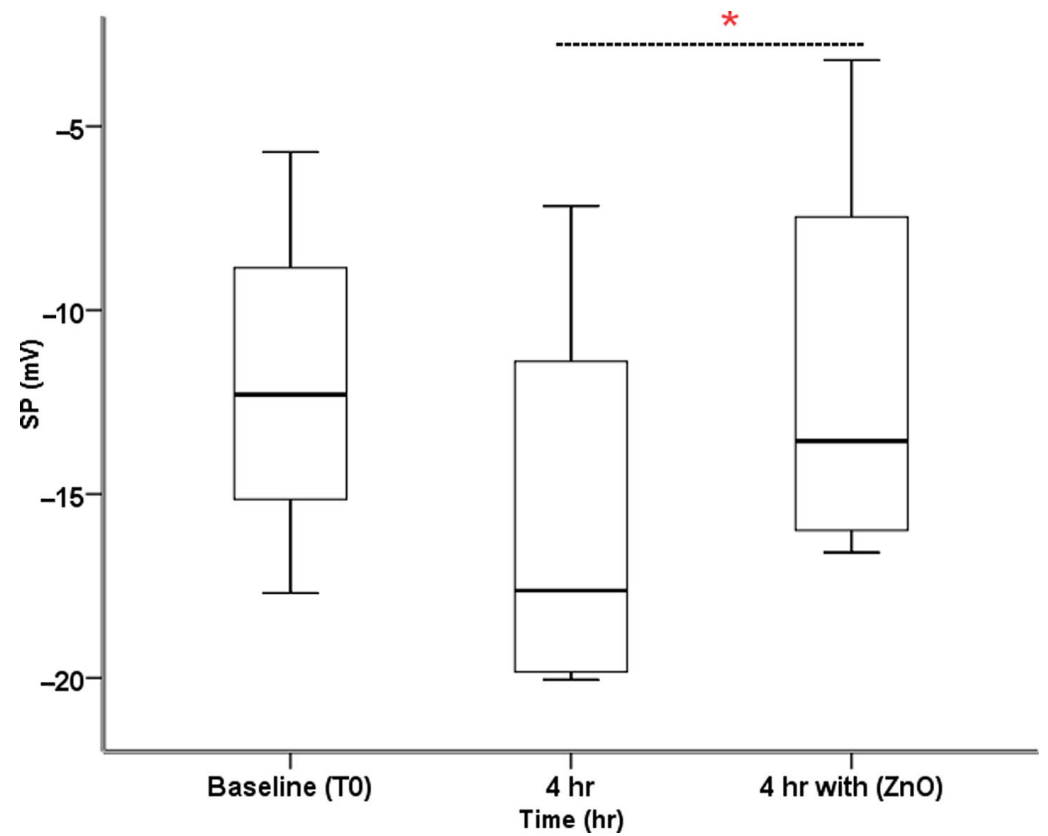

Fig. 3. Impact of wearing gloves for a long duration with and without $\mathrm{ZnO}$ on skin potential (SP). ${ }^{*} \mathrm{p}<0.05$ 


\section{Conclusion}

Hands were the most affected site as a result of adverse skin reactions related to personal protective equipment among healthcare workers during the coronavirus disease outbreak in 2019. Most health care workers wear double gloves to reduce the risk of viral contamination [5]. Long-term use of gloves leads to overhydration of the stratum corneum, which may cause maceration and erosion [1]. This study more specifically addresses the hand skin hydration of health care workers who wear gloves routinely for at least 8 hours a day. The study represents the first attempt at quantifying the hand skin hydration of HCWs by measuring electrodermal activities. Our results demonstrate that the skin conductance and susceptance were remarkably increased by wearing single layer gloves for 4 hours. These results provide evidence to skin surface hydration was increased, whereas both skin conductance and susceptance can quickly and accurately assess the skin hydration condition [6]. This indicates that the water is initiated from the deeper, more hydrated layers of the epidermis and dermis and then moves towards the more superficial SC layer which is considered a mechanism for the transepidermal water loss (TEWL) in the absence of sweat gland activity [7]. Nevertheless, wearing gloves reduces TEWL due to occlusion. This is very similar to normal healthy skin covered by kitchen film, which reduces TEWL and may cause swelling. This suggests that increases in skin SC and SS is due to the decrease in TEWL as a result of skin being covered by gloves. Overall, these findings are in accordance with findings reported by Kawai et al [8] and Mohamad et al [9] which states that the skin SC and SS were significantly and negatively correlated with TEWL. Data also revealed a clear decrease in SP compared to not wearing gloves. This is another important factor in understanding the condition of skin hydration of the hands while wearing gloves. Although the skin surface potential is less affected by hydration, it is highly correlated with TEWL and electrical potential itself might play a crucial role in epidermal barrier homeostasis [10]. This implies that decreased SP is associated with reduced TEWL as a result of wearing gloves.

It was recently reported that in cases where maceration cannot be relieved and exudation occur, hand cream and $\mathrm{ZnO}$ ointment are recommended for HCWs. The Topical $\mathrm{ZnO}$ cream was used as it has a good barrier effect to protect the skin from irritating agents and skin overhydration. The results presented illustrate the effect of the cream on the skin while using gloves for a prolonged period through a decrease in SC, a significant negative decrease in SP, and a slight decrease in SS compared to those wearing gloves without cream. The decrease in both SC and SS can be attributed to the fact that the application of $\mathrm{ZnO}$ provided a notable improvement in TEWL as previously reported [11]. This expected elevate in TEWL may also explain an increase in SP.

The strength of this study is the first attempt at quantifying skin hydration condition for HCWs who are wearing gloves for a long duration due to the COVID-19 pandemic. However, some limitations should also be considered. First, the numbers of participants are small, due to continuous lockdown, along with the difficulty reaching health care workers directly in such circumstances. Secondly, there is a lack of direct measurements of the trans-epidermal water loss by a Tewameter.

In conclusion, remarkable differences were observed in SC, SP and SS for test subjects who wore gloves for four hours when compared to the baseline. Similarly, 
significant differences were noted in each of SP and SS of those wearing gloves during the same period with applying $\mathrm{ZnO}$. The effect of topical $\mathrm{ZnO}$ can be attributed to a change in the water barrier at the top of the stratum corneum of the skin which in turn may alter its hydration and TEWL. The results support the trend of using topical ointment $\mathrm{ZnO}$ after long-term use of gloves to prevent skin complications and also recommend changing the gloves at least every four hours of continuous wearing.

\section{$5 \quad$ Acknowledgment}

The authors would like to thank deeply all the volunteers for their assistance in data collection and who have demonstrated their desire to be the test subjects of this study.

\section{References}

[1] Y. Yan et al., "Consensus of Chinese experts on protection of skin and mucous membrane barrier for health-care workers fighting against coronavirus disease 2019," Dermatol. Ther., vol. 33, no. 4, pp. 1-7, 2020, doi: https://doi.org/10.1111/dth.13310

[2] J. Lan et al., "Skin damage among health care workers managing coronavirus disease-2019," J. Am. Acad. Dermatol., vol. 82, no. 5, pp. 1215-1216, 2020, doi: https://doi.org/10.1016/j. jaad.2020.03.014

[3] D. S. Bari, H. Y. Y. Aldosky, C. Tronstad, H. Kalvøy, and G. Martinsen, "Electrodermal responses to discrete stimuli measured by skin conductance, skin potential, and skin susceptance," Ski. Res. Technol., vol. 24, no. 1, pp. 108-116, 2018, doi: https://doi.org/10.1111/ srt. 12397

[4] D. S. Bari, H. Y. Y. Aldosky, C. Tronstad, H. Kalvøy, and Ø. G. Martinsen, "Electrodermal activity responses for quantitative assessment of felt pain," J. Electr. Bioimpedance, vol. 9, no. 1, pp. 52-58, 2018, doi: https://doi.org/10.2478/joeb-2018-0010

[5] P. Lin et al., "Adverse skin reactions among healthcare workers during the coronavirus disease 2019 outbreak: a survey in Wuhan and its surrounding regions," Br. J. Dermatol., vol. 183, no. 1, pp. 190-192, 2020, doi: https://doi.org/10.1111/bjd.19089

[6] H. Tagami, "Electrical measurement of the hydration state of the skin surface in vivo," Br. J. Dermatol., vol. 171, pp. 29-33, 2014, doi: https://doi.org/10.1111/bjd.13245

[7] E. Corsini and V. Galbiati, "Contact allergy to fragrances: In vitro opportunities for safety assessment," Cosmetics, vol. 6, no. 1, 2019, doi: https://doi.org/10.3390/cosmetics6010019

[8] E. Kawai, J. Nakanishi, N. Kumazawa, K. Ozawa, and M. Denda, "Skin surface electric potential as an indicator of skin condition: A new, non-invasive method to evaluate epidermal condition,” Exp. Dermatol., vol. 17, no. 8, pp. 688-692, 2008, doi: https://doi.org/ $\underline{10.1111 / \mathrm{j} .1600-0625.2007 .00692 . \mathrm{x}}$

[9] M. Mohamad, A. R. Msabbri, and M. Z. Matjafri, "Non invasive measurement of skin hydration and transepidermal water loss in normal skin," CHUSER 2012 - 2012 IEEE Colloq. Humanit. Sci. Eng. Res., no. Chuser, pp. 859-862, 2012, doi: https://doi.org/10.1109/ CHUSER.2012.6504435

[10] E. Kawai, N. Kumazawa, K. Ozawa, and M. Denda, "Skin surface electrical potential as an indicator of skin condition: Observation of surfactant-induced dry skin and middle-aged skin,” Exp. Dermatol., vol. 20, no. 9, pp. 757-759, 2011, doi: https://doi.org/ 10.1111/j.1600-0625.2011.01298.x 
[11] S. Baldwin, M. R. Odio, S. L. Haines, R. J. O’Connor, J. S. Englehart, and A. T. Lane, "Skin benefits from continuous topical administration of a zinc oxide/petrolatum formulation by a novel disposable diaper,” J. Eur. Acad. Dermatology Venereol., vol. 15, no. SUPPL. 1, pp. 5-11, 2001, doi: https://doi.org/10.1046/j.0926-9959.2001.00002.x

\section{Authors}

Haval Y. Yaccob Aldosky is currently Professor at the Department of Physics, College of Science, University of Duhok, Duhok, Kurdistan Region, Iraq. Aldosky received his $\mathrm{PhD}$ degree in 2006 and his research interest includes Electrodermal Activity, Bioimpedance, Biomedical Engineering.

Dindar S. Bari currently works as an Associate professor at the Department of Physics, University of Zakho, Zakho, Kurdistan Region, Iraq. Bari received his PhD degree in 2019. Bari does research in Computational Physics, Medical Radiation Physics and Biomedical Impedance. His current researches are on Electrodermal activity (EDA) recordings.

Ørjan G. Martinsen is a professor of electronics at the Department of Physics, University of Oslo, Norway. He also has an adjunct position as a senior researcher at the Department of Clinical and Biomedical Engineering, Oslo University Hospital, Norway. Martinsen received his PhD degree in 1995 and his research has mainly been focused on the passive, electrical properties of biomaterials. He is the editor-in-chief of the Journal of Electrical Bioimpedance and a co-author of the textbook Bioimpedance and Bioelectricity Basics.

Article submitted 2021-04-20. Resubmitted 2021-05-25. Final acceptance 2021-05-25. Final version published as submitted by the authors. 